\title{
LIST OF PARTICIPANTS OF IAU SYMPOSIUM 194
}

Abazajian, Kevork N., University of California, San Diego, USA Abrahamian, Martin G., Yerevan State Pedagogical Institute, Armenia Alexander, David M., University of Hertfordshire, UK Ali, Gamal Bakr, Ntl Research Inst. of Astron. \& Geophysics, Egypt Amirkhanian, Arthur S., Byurakan Astrophysical Observatory, Armenia Andreasyan, Ruben R., Byurakan Astrophysical Observatory, Armenia Arp, Halton C., Max-Planck-Inst. fur Astrophysik, Garching, Germany Arshakian, Tigran G., Univ. of Cambridge, UK / Byurakan Obs., Armenia Asatrian, Norair S., Byurakan Astrophysical Observatory, Armenia Baan, Willem A., NFRA - Westerbork Observatory, Netherlands Balayan, Smbat K., Byurakan Astrophysical Observatory, Armenia Bergmann, Thaisa S., Instituto de Fisica - UFRGS, Brasil Bertola, Francesco, Dipartimento di Astronomia, Padova Univ., Italy Best, Philip N., Sterrewacht Leiden, Huygens Laboratory, Netherlands Bochkarev, Nikolai G., Sternberg Astronomical Institute, Russia Boyarchuk, Alexandr A., Institute of Astronomy, Moscow, Russia Burenkov, Alexandr N., Special Astrophysical Observatory, Russia Buson, Lucio Maria, Osservatorio di Capodimonte, Napoli, Italy Cappellari, Michele, Dipartimento di Astronomia, Padova Univ., Italy Chatterjee, Tapan K., FCFM, Universidad Autonoma de Puebla, Mexico Clavel, Jean, European Space Agency, Astrophys. Division, Spain Collin-Zahn, Suzy, Observatoire de Paris-Meudon, France Courvoisier, Thierry, INTEGRAL Sci Data Centre, Obs. de Geneve, Switzerland

Cunniffe, John, Dunsink Observatory, Dublin, Ireland

Denissyuk, Eduard K., Astrophysical Institute, Alma-Ata, Kazakhstan Dietrich, Matthias, Landessternwarte Heidelberg, Germany

Dodonov, Serguei N., Special Astrophysical Observatory, Russia Dopita, Michael A., Mt.Stromlo and Siding Springs Observatory, Australia Egikian, Anahit G., Byurakan Astrophysical Observatory, Armenia Fridman, Alexei M., Institute of Astronomy, Moscow, Russia Grigoryan, Levon Sh., Inst. of Applied Problems in Physics, Armenia Gurzadyan, Vahagn G., Yerevan Physics Institute, Armenia Gyulbudaghian, Armen L., Byurakan Astrophysical Observatory, Armenia Gyulzadyan, Marietta V., Byurakan Astrophysical Observatory, Armenia Hagen-Thorn, Vladimir, Sankt-Petersburg State University, Russia Hakopian, Susanna A., Byurakan Astrophysical Observatory, Armenia Harutyunian, Haik A., Byurakan Astrophysical Observatory, Armenia Hirst, Paul, Dept. Physics \& Astronomy, Univ. Leicester, UK Hovhannessian, Martin, Byurakan Astrophysical Observatory, Armenia Huang, Keliang, Physics Department, Nanjing Normal University, China Iskudarian, Sofik G., Byurakan Astrophysical Observatory, Armenia Israelian, Garik L., Instituto de Astrofisica de Canarias, Spain Kaiser, Mary E., Johns Hopkins University, Baltimore, USA Kalloghlian, Arsen T., Byurakan Astrophysical Observatory, Armenia Kandalyan, Rafik A., Byurakan Astrophysical Observatory, Armenia 
Kawaguchi, Toshihiro, Dept of Astronomy, Kyoto University, Japan Kellermann, Kenneth I., National Radio Astronomical Observatory, USA Khachikian, Edward Ye., Byurakan Astrophysical Observatory, Armenia Koratkar, Anuradha P., Space Telescope Science Institute, USA

Kovalev, Yuri Yu., Astronomical Space Centre, Phys. Inst., Moscow, Russia Krolik, Julian H., Johns Hopkins University, Baltimore, USA

Laurent, Olivier, Service d'Astrophysique, Saclay, France

Leeuw, Lerothodi L., Joint Astron.Center \& Univ.of Central Lanchashire, USA

Lelievre, Gerard H., Observatoire de Paris, DASGAL, France

Lominadze, Jumber G., Abastoumani Astrophysical Observatory, Georgia

Longair, Malcolm S., Univ. of Cambridge, Cavendish Laboratory, UK

Lovelace, Richard V.E., Cornell University, Astronomy Dept, USA

Macchetto, F. Duccio, Space Telescope Science Institute, USA

Mahtessian, Abraham P., Byurakan Astrophysical Observatory, Armenia

Malumian, Vigen H., Byurakan Astrophysical Observatory, Armenia

Matveenko, Leonid I., Space Research Institute, RAN, Russia

Melikian, Norair D., Byurakan Astrophysical Observatory, Armenia

Mickaelian, Areg M., Byurakan Astrophysical Observatory, Armenia

Mirabel, I. Felix, Service d'Astrophysique, Saclay, France

Movsessian, Vardan H., Byurakan Astrophysical Observatory, Armenia

Napier, William M., Armagh Observatory, Northern Ireland, UK

Navasardyan, Hripsime, Byurakan Astrophysical Observatory, Armenia

Nikoghossian, Elena H., Byurakan Astrophysical Observatory, Armenia

Notni, Peter, Astrophysikalisches Institut Potsdam, Germany

Ohanian, Gabriel A., Byurakan Astrophysical Observatory, Armenia

Okazaki, Atsuo T., Hokkai-Gakuen University, Japan

Osmer, Patrick S., Ohio State University, Dept of Astronomy, USA

Otterbein, Kai, Landessternwarte Heidelberg, Germany

Panajian, Vazgen G., Byurakan Astrophysical Observatory, Armenia

Petrosian, Artashes R., Byurakan Astrophysical Observatory, Armenia

Petrosian, Vahe, Stanford Univ., Ctr. Sp. Sci \& Astrophysics, USA

Petrov, Georgy T., Institute of Astronomy, Sofia, Bulgaria

Pronik, Iraida I., Crimean Astrophysical Observatory, Ukraine

Robson, E. Ian, Joint Astronomy Centre, Hawaii, USA

Romanova, Marina M., Cornell University, Astronomy Dept, USA

Roscoe, David, Univ. of Sheffield, Dept of Applied Maths, UK

Sadoyan, Avetis H., Yerevan State University, Armenia

Sahakian, Gurgen S., Dept of Theor. Physics, Yerevan State Univ., Armenia

Sambruna, Rita M., Dept of Astronomy, Pennsylvania State Univ., USA

Sanders, David B., Institute for Astronomy, Univ. of Hawaii, USA

Sinanian, Armen A., Byurakan Astrophysical Observatory, Armenia

Smith, H. Eugene, University of California, San Diego, USA

Stepanian, Jivan A., Special Astrophysical Observatory, Russia

Sulentic, Jack W., Univ. of Alabama, Dept of Physics \& Astron., USA

Terzian, Yervant, Cornell University, Dept of Astronomy, USA

Tran, Hien D., Inst. of Geophys. and Planetary Physics, USA

Trevese, Dario, Istituto Astronomico, Universita di Roma, Italy

Turatto, Massimo, Osservatorio Astronomico di Padova, Italy 
Vardanyan, Rafik A., Byurakan Astrophysical Observatory, Armenia

Vardapetian, Ruben, INTAS, Brussels, Belgium

Veron, Marie-Paule, Observatoire de Haute-Provence, France

Veron, Philippe, Observatoire de Haute-Provence, France

Weedman, Daniel W., Pennsylvania State Univ, Dept of Astron Astrophys, USA

Xanthopoulos, Emily, NRAL Jodrell Bank, UK

Zahn, Jean-Paul, Observatoire de Paris-Meudon, France

Zasov, Anatoly V., Sternberg Astronomical Institute, Moscow, Russia 